\title{
The relations between bi-periodic jacobsthal and bi-periodic jacobsthal lucas sequence
}

\author{
Şükran UYGUN ${ }^{1, *(i D)}$ \\ ${ }^{I}$ Gaziantep University, Faculty of Science And Arts, Department of Mathematics, Gaziantep / TURKEY
}

\begin{abstract}
In this paper, one of the special integer sequences, Jacobsthal and Jacobsthal Lucas sequences which are encountered in computer science is generalized according to parity of the index of the entries of the sequences, called bi-periodic Jacobsthal and Jacobsthal Lucas sequences. The definitions of the bi-periodic Jacobsthal and Jacobsthal Lucas sequences are given by using classic Jacobsthal and Jacobsthal Lucas sequences. In literature, there were some relations for the bi-periodic Jacobsthal and Jacobsthal Lucas sequences. We find new identities for these sequences. If we substitute $a=b=1$ in the results, we get identities for classic Jacobsthal and Jacobsthal Lucas sequences.
\end{abstract}

\section{Article info}

History:

Received: 16.07 .2020

Accepted: 02.06.2021

Keywords:

Bi-periodic Jacobsthal sequence,

Generalized Jacobsthal Lucas sequence,

Binet formula.

\section{Introduction}

The classical Jacobsthal sequence is defined as $j_{n}=$ $j_{n-1}+2 j_{n-2}$ with initial conditions $j_{0}=0, \quad j_{1}=$ 1 and the Jacobsthal Lucas sequence is defined as with the initial conditions $c_{0}=2, c_{1}=1$ [1]. There are many generalizations on special integer sequences. With this in mind, we proceed with the introduction as follows. In the year 2009, a paper entitled, the generalized Fibonacci sequence has been published by Edson and Yayenie [2,3]. Jun, Choi gave some properties of the bi-periodic Fibonacci sequence by using a special matrix in [4]. Bilgici [5] introduced the bi-periodic Lucas sequence into literature in 2014. Uygun and Owusu demonstrated a new generalization for the Jacobsthal sequence called bi-periodic Jacobsthal sequence in [6]. The authors evaluated some relations for bi-periodic Jacobsthal sequence in [7]. In [8], Uygun and Owusu demonstrated the bi-periodic Jacobsthal Lucas sequence. Uygun, Karatas defined a new generalization of Pell-Lucas numbers which is called bi-periodic Pell-Lucas sequence in [9]. Choo studied some identities of generalized bi-periodic Fibonacci sequence in [10]. Gul studied bi-periodic Jacobsthal and Jacobsthal-Lucas quaternions in [11]. Komatsu, Ramírez studied on convolutions of the biperiodic Fibonacci numbers in [12].

For every integer $n$, any nonzero real numbers $a, b$ the generalized Jacobsthal sequence $\left\{J_{m}\right\}_{m=0}^{\infty}$ and the generalized Jacobsthal Lucas sequence $\left\{C_{m}\right\}_{m=0}^{\infty}$ satisfy the following equations.
$J_{n}=\left\{\begin{array}{ll}a J_{n-1}+2 J_{n-2} & \text { if } n \text { is even } \\ b J_{n-1}+2 J_{n-2} & \text { if } n \text { is odd }\end{array} \quad(n \geq 2)\right.$

with initial values $J_{0}=0$ and $J_{1}=1$.

$C_{n}=\left\{\begin{array}{ll}b C_{n-1}+2 C_{n-2} & \text { if } n \text { is even } \\ a C_{n-1}+2 C_{n-2} & \text { if } n \text { is odd }\end{array} \quad(n \geq 2)\right.$

with initial values, $C_{0}=2$ and $C_{1}=a$ in $[6,8]$.

If we take $a=b=1$ then we have the classic Jacobsthal and classic Jacobsthal Lucas sequences respectively.

The sequence $\left\{J_{n}\right\}_{n=0}^{\infty}$ satisfies the following properties

$J_{2 n}=(a b+4) J_{2 n-2}-4 J_{2 n-4}$

$J_{2 n+1}=(a b+4) J_{2 n-1}-4 J_{2 n-3}$.

The sequence $\left\{C_{n}\right\}_{n=0}^{\infty}$ also satisfies the following properties

$$
\begin{aligned}
& C_{2 n}=(a b+4) C_{2 n-2}-4 C_{2 n-4} \\
& C_{2 n+1}=(a b+4) C_{2 n-1}-4 C_{2 n-3}
\end{aligned}
$$

For every $n$ belonging to the set of natural numbers, the Binet formula for the bi-periodic Jacobsthal sequence is given by 
$J_{n}=\frac{a^{1-\xi(n)}}{(a b)^{\left\lfloor\frac{n}{2}\right\rfloor}}\left(\frac{\alpha^{n}-\beta^{n}}{\alpha-\beta}\right)$

where

$\alpha=\frac{a b+\sqrt{a^{2} b^{2}+8 a b}}{2}$ and $\quad \beta=\frac{a b-\sqrt{a^{2} b^{2}+8 a b}}{2}$

are the roots of the characteristic polynomial given by $x^{2}-a b x-2 a b=0$. Similarly, Binet formula for the bi-periodic Jacobsthal Lucas sequence is given by

$C_{n}=\frac{a^{\xi(n)}}{(a b)^{\left\lfloor\frac{n+1}{2}\right\rfloor}}\left(\alpha^{n}+\beta^{n}\right)$

The authors evaluated some relations of bi-periodic Jacobsthal sequence in [7] as:

a) $\alpha^{n}=a^{\left\lfloor\frac{n}{2}\right\rfloor-\xi(n-1)} b^{\left\lfloor\frac{n}{2}\right\rfloor} J_{n} \alpha+2 a^{\left\lfloor\frac{n}{2}\right\rfloor} b^{\left\lfloor\frac{n}{2}\right\rfloor+\xi(n)} J_{n-1}$

b) $\beta^{n}=a^{\left\lfloor\frac{n}{2}\right\rfloor-\xi(n-1)} b^{\left\lfloor\frac{n}{2}\right\rfloor} J_{n} \beta+2 a^{\left\lfloor\frac{n}{2}\right\rfloor} b^{\left\lfloor\frac{n}{2}\right\rfloor+\xi(n)} J_{n-1}$

c) $J_{n+6}=(a b+6) a^{1-\xi(n)} b^{\xi(n)} J_{n+3}-8 J_{n}$

d) $a J_{2 n-1}=J_{n+1} J_{n}+J_{n-1} J_{n-2}$

e) $J_{m+n-1}=\left(\frac{b}{a}\right)^{1-\xi(m n+n-m)} J_{m} J_{n}+2\left(\frac{b}{a}\right)^{\xi(m n)} J_{m-1} J_{n-1}$

f) $J_{2 m-1}=\left(\frac{b}{a}\right)^{\xi(m+1)}\left(J_{m}\right)^{2}+2\left(\frac{b}{a}\right)^{\xi(m)}\left(J_{m-1}\right)^{2}$

g) $J_{n} J_{n+2}=\left(\frac{a}{b}\right)^{\xi(n+1)}\left[\left(\frac{b}{a}\right)^{\xi(n)} J_{n+1}^{2}-(-2)^{n+1}\right]$

h) $J_{m}=a^{\xi(m-1)} \sum_{k=0}^{\left[\frac{m-1}{2}\right\rfloor}\left(\begin{array}{c}m-k-1 \\ k\end{array}\right)(a b)^{\left\lfloor\frac{m-1}{2}\right\rfloor-k} 2^{k}$

i) $J_{m}=\frac{a^{\xi(m+1)}}{2^{m-1}} \sum_{k=0}^{\left[\frac{m-1}{2}\right\rfloor}\left(\begin{array}{c}m \\ 2 k+1\end{array}\right)(a b)^{\left\lfloor\frac{m-1}{2}\right\rfloor-k}(a b+8)^{k}$

The authors evaluated some relations between the bi-periodic Jacobsthal sequence and the bi-periodic Jacobsthal Lucas sequences for all integers $m$ and $n$ in as:

a) $(a b+8) J_{m}=2 C_{m-1}+C_{m+1}$

b) $J_{m+n}=\frac{1}{2}\left[\left(\frac{b}{a}\right)^{\xi(m+1) \xi(n)} J_{m} C_{n}+\left(\frac{b}{a}\right)^{\xi(m) \xi(n+1)} J_{n} C_{m}\right]$

c) $J_{m-n}=\frac{(-1)^{n}}{2^{n+1}}\left[\left(\frac{b}{a}\right)^{\xi(m+1) \xi(n)} J_{m} C_{n}-\left(\frac{b}{a}\right)^{\xi(m) \xi(n+1)} J_{n} C_{m}\right]$

d) $C_{m+n}=\frac{1}{2}\left[\left(a^{2} b^{2}+8 a b\right)\left(\frac{1}{a^{2}}\right)^{\xi(m+1) \xi(n+1)}\left(\frac{1}{a b}\right)^{1-\xi(m+1) \xi(n+1)} J_{m} J_{n}+\left(\frac{b}{a}\right)^{\xi(m) \xi(n)} C_{m} C_{n}\right]$ 
e) $C_{m-n}=\frac{(-1)^{n}}{2^{n+1}}\left[\begin{array}{c}\left(\frac{b}{a}\right)^{\xi(m) \xi(n)} C_{m} C_{n} \\ -\left(a^{2} b^{2}+8 a b\right)\left(\frac{1}{a^{2}}\right)^{\xi(m+1) \xi(n+1)}\left(\frac{1}{a b}\right)^{1-\xi(m+1) \xi(n+1)} \\ J_{m} J_{n}\end{array}\right]$

f) $J_{n+1}=\frac{1}{2}\left(C_{n}+a^{\xi(n)} b^{\xi(n+1)} J_{n}\right)$

g) $\quad C_{n+1}=\frac{1}{2}\left[(a b+8) J_{n}+a^{\xi(n+1)} b^{\xi(n)} C_{n}\right]$

h) $\left(\frac{b}{a}\right)^{\xi(n)} C_{n}{ }^{2}-\left(a^{2} b^{2}+8 a b\right)\left(\frac{1}{a^{2}}\right)^{\xi(n+1)}\left(\frac{1}{a b}\right)^{\xi(n)} J_{n}{ }^{2}=4(-2)^{n}$

i) $C_{2 n}=\frac{1}{2}\left[\left(a^{2} b^{2}+8 a b\right)\left(\frac{1}{a^{2}}\right)^{\xi(n+1)}\left(\frac{1}{a b}\right)^{\xi(n)} J_{n}{ }^{2}+\left(\frac{b}{a}\right)^{\xi(n)} C_{n}{ }^{2}\right]$

j) $\quad C_{2 m} C_{2 n}=C_{2 m+2 n}+4^{m} C_{2 n-2 m}$

k) $C_{2 m} C_{2 n}=\left(\frac{b}{a}\right)^{\xi(m+n)}\left[C_{m+n}^{2}+(2)^{2 n} C^{2}{ }_{m-n}\right]-4(-2)^{m+n}$

1) $C_{2 m} C_{2 n}=a^{-2 \xi(m+n+1)}(a b)^{-\xi(m+n)}\left(a^{2} b^{2}+8 a b\right) J_{m+n}^{2}+2^{2 n}\left(\frac{b}{a}\right)^{\xi(m+n)} C_{m-n}^{2}$

m) $C_{2 m} C_{2 n}=a^{-2 \xi(m+n+1)}(a b)^{-\xi(m+n)}\left(a^{2} b^{2}+8 a b\right) J^{2}{ }_{m+n}+2^{2 n}\left(\frac{b}{a}\right)^{\xi(m+n)} C^{2}{ }_{m-n}$

n) $C_{2 m} C_{2 n}=2^{2 n} a^{-2 \xi(m+n+1)}(a b)^{-\xi(m+n)}\left(a^{2} b^{2}++8 a b\right) J^{2}{ }_{m-n}\left(\frac{b}{a}\right)^{\xi(m+n)} C^{2}{ }_{m+n}$

o) $C_{n} C_{n+1}=C_{2 n+1}+(-2)^{n} a$

p) $2\left(\frac{b}{a}\right)^{\xi(n)} C_{n}^{2}+\left(\frac{b}{a}\right)^{\xi(n+1)} C_{n+1}^{2}=(a b+8) J_{2 n+1}$

\section{Main Results}

\subsection{New properties between the bi-periodic jacobsthal sequence and the bi-periodic jacobsthal lucas sequences}

Jacobsthal numbers have applications in such areas as tiling, graph matching, alternating sign matrices, etc. [13-16]. So, in this part we want to develop this number sequence and find new properties of the sequence.

Theorem 2.1 For any integers $m$ and $n$, we have

a) $J_{3 n}=J_{n}\left[C_{2 n}+(-2)^{n}\right]$,

b) $J_{3 n}=\left(\frac{b}{a}\right)^{\xi(n)}\left[J_{2 n} C_{n}-(-2)^{n} J_{n}\right]$.

Proof: For the proof of a) we use Binet formula for biperiodic Jacobsthal sequence and bi-periodic Jacobsthal Lucas sequences

$$
J_{n} C_{2 n}=\left(\frac{a^{1-\xi(n)}}{(a b)^{\left.\frac{n}{2}\right\rfloor}}\right)\left(\frac{a^{\xi(2 n)}}{\left.(a b)^{\frac{2 n+1}{2}}\right\rfloor}\right)\left(\alpha^{2 n}+\beta^{2 n}\right)\left(\frac{\alpha^{n}-\beta^{n}}{\alpha-\beta}\right)
$$

$$
\begin{aligned}
& =\frac{a^{\xi(n+1)}}{(a b)^{\left[\frac{n}{2}\right]+\left[\frac{2 n+1}{2}\right]} \frac{1}{\alpha-\beta}}\left(\alpha^{3 n}-\beta^{3 n}+\alpha^{n} \beta^{2 n}-\right. \\
& \alpha^{2 n} \beta^{n} \\
& =\frac{a^{\xi(n+1)}}{(a b)^{\left[\frac{3 n}{2}\right]}} \frac{1}{\alpha-\beta}\left(\alpha^{3 n}-\beta^{3 n}-\alpha^{n} \beta^{n}\left(\alpha^{n}-\beta^{n}\right)\right)
\end{aligned}
$$

$$
=\frac{a^{\xi(n+1)}}{(a b)^{\left[\frac{3 n}{2}\right]}} \frac{\alpha^{3 n}-\beta^{3 n}}{\alpha-\beta}-\frac{a^{\xi(n+1)}}{(a b)^{\left\lfloor\frac{3 n}{2}\right]}}(-2 a b)^{n} \frac{\alpha^{n}-\beta^{n}}{\alpha-\beta}
$$

$$
\begin{aligned}
& =\frac{a^{\xi(n+1)}}{(a b)^{\left\lfloor\frac{3 n}{2}\right\rfloor}} \frac{\alpha^{3 n}-\beta^{3 n}}{\alpha-\beta}-(-2)^{n} \frac{a^{1-\xi(n)}}{(a b)^{\left\lfloor\frac{n}{2}\right\rfloor}} \frac{\alpha^{n}-\beta^{n}}{\alpha-\beta} \\
& =J_{3 n}-(-2)^{n} J_{n}
\end{aligned}
$$


For the proof of $b$ )

$$
\begin{aligned}
& J_{2 n} C_{n}=\left(\frac{a^{1-\xi(2 n)}}{(a b)^{n}}\right)\left(\frac{a^{\xi(n)}}{(a b)^{\left\lfloor\frac{n+1}{2}\right\rfloor}}\right)\left(\alpha^{n}+\beta^{n}\right)\left(\frac{\alpha^{2 n}-\beta^{2 n}}{\alpha-\beta}\right) \\
& =\frac{a^{1+\xi(n)}}{(a b)^{n+\left\lfloor\frac{n+1}{2}\right\rfloor}} \frac{1}{\alpha-\beta}\left(\alpha^{3 n}-\beta^{3 n}+\alpha^{2 n} \beta^{n}-\alpha^{n} \beta^{2 n}\right)
\end{aligned}
$$

If $n$ is odd, we have

$J_{2 n} C_{n}=\frac{a^{2}}{(a b)^{\left\lfloor\frac{3 n}{2}\right\rfloor+1}} \frac{1}{\alpha-\beta}\left(\alpha^{3 n}-\beta^{3 n}+\alpha^{n} \beta^{n}\left(\alpha^{n}-\right.\right.$ $\left.\left.\beta^{n}\right)\right)$

$$
\begin{aligned}
& =\frac{a}{b} \frac{a b}{(a b)^{\left\lfloor\frac{3 n}{2}\right\rfloor+1}} \frac{1}{\alpha-\beta}\left(\alpha^{3 n}-\beta^{3 n}+\right. \\
& (-2 a b)^{n}\left(\alpha^{n}-\right. \\
& \left.\left.\beta^{n}\right)\right) \\
& =\frac{a}{b} \frac{1}{(a b)^{\left\lfloor\frac{3 n}{2}\right\rfloor}} \frac{\alpha^{3 n}-\beta^{3 n}}{\alpha-\beta}+ \\
& \frac{a}{b} \frac{1}{(a b)^{\left\lfloor\frac{3 n}{2}\right\rfloor-n}}(-2)^{n}\left(\frac{\alpha^{n}-\beta^{n}}{\alpha-\beta}\right) \\
& =\frac{a}{b} \frac{1}{(a b)^{\left\lfloor\frac{3 n}{2}\right\rfloor}} \frac{\alpha^{3 n}-\beta^{3 n}}{\alpha-\beta}+\frac{a}{b}(-2)^{n} \frac{1}{(a b)^{\left\lfloor\frac{n}{2}\right\rfloor}}\left(\frac{\alpha^{n}-\beta^{n}}{\alpha-\beta}\right) \\
& =\frac{a}{b} J_{3 n}+\frac{a}{b}(-2)^{n} J_{n}
\end{aligned}
$$

Now if $n$ is even, we have

$$
\begin{aligned}
J_{2 n} C_{n} & =\frac{a}{(a b)^{\left\lfloor\frac{3 n}{2}\right\rfloor}} \frac{1}{\alpha-\beta}\left(\alpha^{3 n}-\beta^{3 n}-\alpha^{n} \beta^{n}\left(\alpha^{n}-\beta^{n}\right)\right) \\
& =\frac{a}{(a b)^{\left\lfloor\frac{3 n}{2}\right\rfloor}} \frac{1}{\alpha-\beta}\left(\alpha^{3 n}-\beta^{3 n}-(-2 a b)^{n}\left(\alpha^{n}-\right.\right. \\
& \left.\left.\beta^{n}\right)\right) \\
& =\frac{a}{(a b)^{\left\lfloor\frac{3 n}{2}\right\rfloor}} \frac{\alpha^{3 n}-\beta^{3 n}}{\alpha-\beta}-\frac{a}{(a b)^{\left\lfloor\frac{3 n}{2}\right\rfloor-n}}(-2)^{n}\left(\frac{\alpha^{n}-\beta^{n}}{\alpha-\beta}\right) \\
& =\frac{a}{(a b)^{\left\lfloor\frac{3 n}{2}\right\rfloor}} \frac{\alpha^{3 n}-\beta^{3 n}}{\alpha-\beta}-(-2)^{n} \frac{a}{(a b)^{\left\lfloor\frac{n}{2}\right\rfloor}}\left(\frac{\alpha^{n}-\beta^{n}}{\alpha-\beta}\right) \\
& =J_{3 n}-(-2)^{n} J_{n}
\end{aligned}
$$

By condensing the result gives us the desired result.

Now taking $a=b=1$ into Theorem 2.1 gives

$j_{3 n}=j_{n}\left[c_{2 n}+(-2)^{n}\right]=j_{2 n} c_{n}+(-2)^{n} j_{n}$.

Theorem 2.2 For any integers $m$ and $n$, we have the following property for bi-periodic Jacobsthal sequence and bi-periodic Jacobsthal Lucas sequences

$J_{2 m+n}=\left(\frac{b}{a}\right)^{\xi(m+1) \xi(n)} J_{m} C_{m+n}+(-2)^{m} J_{n}$.

\section{Proof:}

$$
\begin{aligned}
J_{m} C_{m+n} & =\left(\frac{a^{1-\xi(m)}}{(a b)^{\left\lfloor\frac{m}{2}\right\rfloor}}\right)\left(\frac{a^{\xi(m+n)}}{(a b)^{\left\lfloor\frac{m+n+1}{2}\right\rfloor}}\right)\left(\alpha^{m+n}+\beta^{m+n}\right)\left(\frac{\alpha^{m}-\beta^{m}}{\alpha-\beta}\right) \\
& =\frac{a^{1-\xi(m)+\xi(m+n)}}{(a b)^{\left\lfloor\frac{m}{2}\right\rfloor+\left\lfloor\frac{m+n+1}{2}\right\rfloor} \frac{\alpha^{2 m+n}-\beta^{2 m+n}+\alpha^{m} \beta^{m+n}-\alpha^{m+n} \beta^{m}}{\alpha-\beta}}
\end{aligned}
$$

If both $m$ and $n$ are even, $m+n$ is even as well and hence we have

$$
\begin{aligned}
J_{m} C_{m+n} & =\frac{a}{(a b)^{\left\lfloor\frac{2 m+n}{2}\right\rfloor}} \frac{\alpha^{2 m+n}-\beta^{2 m+n}-\alpha^{m} \beta^{m}\left(\alpha^{n}-\beta^{n}\right)}{\alpha-\beta} \\
& =\frac{a}{(a b)^{\left\lfloor\frac{2 m+n}{2}\right\rfloor}} \frac{\alpha^{2 m+n}-\beta^{2 m+n}}{\alpha-\beta}-(-2 t)^{m} \frac{a}{(a b)^{\left\lfloor\frac{2 m+n}{2}\right\rfloor-m}} \frac{\alpha^{n}-\beta^{n}}{\alpha-\beta} \\
& =J_{2 m+n}-(-2)^{m} J_{n}
\end{aligned}
$$

If $m$ and $n$ are both odd, $m+n$ even and hence we have

$J_{m} C_{m+n}=\frac{1}{(a b)^{\left\lfloor\frac{2 m+n}{2}\right\rfloor}} \frac{\alpha^{2 m+n}-\beta^{2 m+n}+\alpha^{m} \beta^{m+n}-\alpha^{m+n} \beta^{m}}{\alpha-\beta}$ 


$$
\begin{aligned}
& =\frac{1}{(a b)\left\lfloor\frac{2 m+n}{2}\right\rfloor} \frac{\alpha^{2 m+n}-\beta^{2 m+n}}{\alpha-\beta}-(-2)^{m} \frac{1}{(a b)^{\left.\frac{2 m+n}{2}\right\rfloor-m}}\left(\frac{\alpha^{n}-\beta^{n}}{\alpha-\beta}\right) \\
& =J_{2 m+n}-(-2)^{m} J_{n}
\end{aligned}
$$

If $m$ is odd and $n$ is even, then $m+n$ is odd and hence we have

$$
\begin{aligned}
J_{m} C_{m+n} & =\frac{a}{(a b)^{\left\lfloor\frac{2 m+n}{2}\right\rfloor}} \frac{1}{\alpha-\beta}\left(\alpha^{2 m+n}-\beta^{2 m+n}+\alpha^{m} \beta^{m+n}-\alpha^{m+n} \beta^{m}\right) \\
& =\frac{a}{(a b)^{\left.\mid \frac{2 m+n}{2}\right\rfloor}} \frac{1}{\alpha-\beta}\left(\alpha^{2 m+n}-\beta^{2 m+n}-\alpha^{m} \beta^{m}\left(\alpha^{n}-\beta^{n}\right)\right) \\
& =\frac{a}{(a b)^{\left.\mid \frac{2 m+n}{2}\right\rfloor}} \frac{\alpha^{2 m+n}-\beta^{2 m+n}}{\alpha-\beta}-\frac{(-2)^{m} a}{(a b)^{\left.\frac{2 m+n}{2}\right\rfloor-m}}\left(\frac{\alpha^{n}-\beta^{n}}{\alpha-\beta}\right) \\
& =J_{2 m+n}-(-2)^{m} J_{n}
\end{aligned}
$$

Finally if $m$ is even and $n$ is odd, $m+n$ is odd

$$
\begin{aligned}
J_{m} C_{m+n} & =\frac{a^{2}}{(a b)^{\left.\right|^{\frac{2 m+n}{2}}++1}} \frac{\alpha^{2 m+n}-\beta^{2 m+n}+\alpha^{m} \beta^{m+n}-\alpha^{m+n} \beta^{m}}{\alpha-\beta} \\
& =\frac{a}{b} \frac{a b}{(a b)^{\left.\frac{2 m+n}{2}\right\rfloor+1}} \frac{\alpha^{2 m+n}-\beta^{2 m+n}-\alpha^{m} \beta^{m}\left(\alpha^{n}-\beta^{n}\right)}{\alpha-\beta} \\
& =\frac{a}{b} \frac{1}{(a b)^{\mid \frac{2 m+n}{2}}} \frac{\alpha^{2 m+n}-\beta^{2 m+n}}{\alpha-\beta}-\frac{a}{b}(-2)^{m} \frac{1}{(a b)^{\left.\mid \frac{2 m+n}{2}\right]-m}}\left(\frac{\alpha^{n}-\beta^{n}}{\alpha-\beta}\right) \\
& =\frac{a}{b} J_{2 m+n}-\frac{a}{b}(-2)^{m} J_{n} .
\end{aligned}
$$

Therefore, condensing the above with the help of the parity function gives

$J_{2 m+n}=\left(\frac{b}{a}\right)^{\xi(m+1) \xi(n)} J_{m} C_{m+n}+(-2)^{m} J_{n}$

which completes the proof.

Now taking $a=b=1$ into Theorem 2.2 gives

$j_{2 m+n}=j_{m} c_{m+n}+(-2)^{m} j_{n}$.

Theorem 2.3 The following identity is satisfied by bi-periodic Jacobsthal sequence and bi-periodic Jacobsthal Lucas sequence for any integer $n$

$$
(\alpha-\beta)^{2} J_{2 n+3} J_{2 n-3}=C_{4 n}-(-2)^{2 n-3} C_{6} .
$$

Proof: By (1) and (2), we establish

$$
\begin{aligned}
& J_{2 n+3} J_{2 n-3}=\left(\frac{a^{1-\xi(2 n+3)}}{\left.(a b)^{\frac{2 n+3}{2}}\right\rfloor} \frac{\alpha^{2 n+3}-\beta^{2 n+3}}{\alpha-\beta}\right)\left(\frac{a^{1-\xi(2 n-3)}}{(a b)^{\left.\frac{2 n-3}{2}\right\rfloor}} \frac{\alpha^{2 n-3}-\beta^{2 n-3}}{\alpha-\beta}\right) \\
& =\frac{1}{\left.(a b) \frac{2 n+3}{2}\right\rfloor+\left[\frac{2 n-3}{2}\right\rfloor} \frac{\alpha^{4 n}+\beta^{4 n}-\alpha^{2 n-3} \beta^{2 n-3}\left(\alpha^{6}+\beta^{6}\right)}{(\alpha-\beta)^{2}} .
\end{aligned}
$$


Multiplying through by $(\alpha-\beta)^{2}$ gives the following

$$
\begin{aligned}
(\alpha-\beta)^{2} J_{2 n+3} J_{2 n-3} & =\frac{\alpha^{4 n}+\beta^{4 n}-(-2 a b)^{2 n-3}\left(\alpha^{6}+\beta^{6}\right)}{(a b)^{2 n}} \\
& =\frac{a^{\xi(4 n)}}{(a b)^{\left[\frac{4 n+1}{2}\right]}}\left(\alpha^{4 n}+\beta^{4 n}\right)-(-2)^{2 n-3} \frac{a^{\xi(6)}}{(a b)^{\left[\frac{6+1}{2}\right]}}\left(\alpha^{6}+\beta^{6}\right) \\
& =C_{4 n}-(-2)^{2 n-3} C_{6}
\end{aligned}
$$

which completes the proof.

Now taking $a=b=1$ into Theorem 2.3 gives

$9 j_{2 n+3} j_{2 n-3}=c_{4 n}-(-2)^{2 n-3} c_{6}$.

Theorem 2.4 For any integer $n$, we have for bi-periodic Jacobsthal sequence and bi-periodic Jacobsthal Lucas sequences

$2 J_{2 n+1}=\left(\frac{b}{a}\right)^{\xi(n)} J_{n+1} C_{n+2}-b J_{n+2} C_{n}+(-2)^{n}(a b-2)$.

\section{Proof:}

$$
\begin{aligned}
&\left(\frac{b}{a}\right)^{\xi(n)} J_{n+1} C_{n+2}=\left(\frac{b}{a}\right)^{\xi(n)}\left(\frac{a^{\xi(n)}}{(a b)^{\left[\frac{n+1}{2}\right]}} \frac{\alpha^{n+1}-\beta^{n+1}}{\alpha-\beta}\right)\left(\frac{a^{\xi(n+2)}\left(\alpha^{n+2}+\beta^{n+2}\right)}{(a b)^{\left[\frac{n+3}{2}\right\rfloor}}\right) \\
&=\left(\frac{b}{a}\right)^{\xi(n)}\left(\frac{a^{2 \xi(n)}}{(a b)^{2}\left[\frac{n+1}{2}\right\rfloor+1} \frac{\alpha^{2 n+3}-\beta^{2 n+3}-(\alpha \beta)^{n+1}(\alpha-\beta)}{\alpha-\beta}\right) \\
&=\left(\frac{(a b) \xi(n)}{(a b)^{n+1+\xi(n)}} \frac{\alpha^{2 n+3}-\beta^{2 n+3}}{\alpha-\beta}\right)-\frac{(2 a b)^{n+1}(\alpha-\beta)}{(a b)^{n+1}} \\
&=J_{2 n+3}-(-2)^{n+1} \\
& b J_{n+2} C_{n}= b\left(\frac{a^{1-\xi(n+2)}}{(a b)^{\left.\frac{n+2}{2}\right]}} \frac{\alpha^{n+2}-\beta^{n+2}}{\alpha-\beta}\right)\left(a^{\xi(n)} \frac{\alpha^{n}+\beta^{n}}{(a b)^{\left.\frac{n+1}{2}\right]}}\right) \\
&=\left(\frac{a b}{(a b)^{n+1}} \frac{\alpha^{2 n+2}-\beta^{2 n+2}+(\alpha \beta)^{n}\left(\alpha^{2}-\beta^{2}\right)}{\alpha-\beta}\right) \\
&=b\left(\frac{a^{1-\xi(2 n+2)}}{(a b)^{n+1}} \frac{\alpha^{2 n+2}-\beta^{2 n+2}}{\alpha-\beta}\right)+\frac{(-2 a b)^{n} a b(\alpha+\beta)}{(a b)^{n+1}} \\
&=b J_{2 n+2}+(-2)^{n} \mathrm{ab}
\end{aligned}
$$

Therefore we have

$$
\begin{aligned}
& \left(\frac{b}{a}\right)^{\xi(n)} J_{n+1} C_{n+2}-b J_{n+2} C_{n}+(-2)^{n}(a b-2) \\
& =J_{2 n+3}-(-2)^{n+1}-b J_{2 n+2}-(-2)^{n} \mathrm{ab}+(-2)^{n}(a b-2) \\
& =J_{2 n+3}-b J_{2 n+2}=2 J_{2 n+1} .
\end{aligned}
$$

Now taking $a=b=1$ into Theorem 2.4 gives 
$2 j_{2 n+1}=j_{n+1} c_{n+2}-j_{n+2} c_{n}-(-2)^{n}$.

Theorem 2.5 For any $n, n$th element of bi-periodic Jacobsthal Lucas sequence is demonstrated by the following equality:

$C_{n}=\left(\frac{b}{a}\right)^{\xi(n+1) \xi(m)} C_{m} J_{n-m+1}+2\left(\frac{b}{a}\right)^{\xi(n+1) \xi(m+1)} C_{m-1} J_{n-m}$.

Proof: For proof we use the following properties

$\frac{1}{2}(\xi(n)+\xi(m)-\xi(m+n))=\xi(n) \xi(m)$

$n-\xi(n)=2\left\lfloor\frac{n}{2}\right\rfloor$

By Binet formulas $(1,2)$, we have

$$
\begin{aligned}
& \left(\frac{b}{a}\right)^{\xi(n+1) \xi(m)} C_{m} J_{n-m+1}+2\left(\frac{b}{a}\right)^{\xi(n+1) \xi(m+1)} C_{m-1} J_{n-m} \\
& =\left(\frac{b}{a}\right)^{\frac{1}{2}(\xi(n+1)+\xi(m)-\xi(m+n+1))}\left(\frac{a^{\xi(m)}}{(a b)^{\left.\frac{m+1}{2}\right]}}\left(\alpha^{m}+\beta^{m}\right)\right)\left(\frac{a^{1-\xi(n-m+1)}}{\left.(a b)\right|^{\left.\frac{n-m+1}{2}\right]}} \frac{\alpha^{n-m+1}-\beta^{n-m+1}}{\alpha-\beta}\right)+ \\
& 2\left(\frac{b}{a}\right)^{\frac{1}{2}(\xi(n+1)+\xi(m+1)-\xi(m+n+2))}\left(\frac{a^{\xi(m-1)}}{(a b)^{\left.\frac{m}{2}\right\rfloor}}\left(\alpha^{m-1}+\beta^{m-1}\right)\right)\left(\frac{a^{1-\xi(n-m)}}{(a b)^{\left.\frac{n-m}{2}\right]}} \frac{\alpha^{n-m}-\beta^{n-m}}{\alpha-\beta}\right)
\end{aligned}
$$

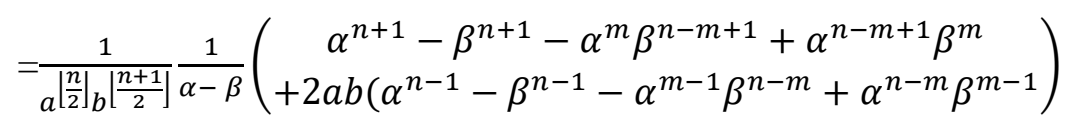

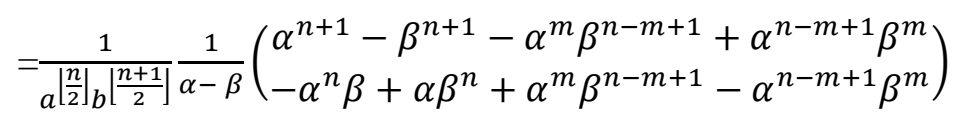

After simplifications we have

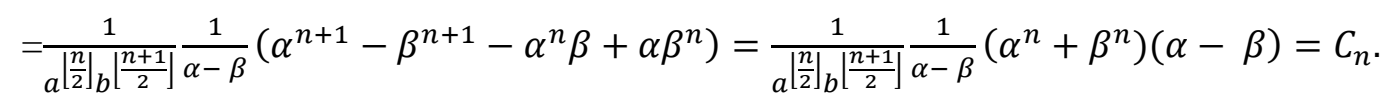

Now taking $a=b=1$ into Theorem 2.5 gives

$c_{n}=c_{m} j_{n-m+1}+2 c_{m-1} j_{n-m}$.

Theorem 2.6 For any $m, n$ the $m n$th element of bi-periodic Jacobsthal sequence is given by

$J_{m n}=\left(\frac{b}{a}\right)^{\xi(n) \xi(m)} C_{m} J_{m(n-1)}-(-2)^{m} J_{m(n-2)}$.

Proof: By using the following property,

$\xi(n) \xi(m)=\left\lfloor\frac{m+n}{2}\right\rfloor-\left\lfloor\frac{m}{2}\right\rfloor-\left\lfloor\frac{n}{2}\right\rfloor$

we have

$$
\left(\frac{b}{a}\right)^{\xi(n) \xi(m)} C_{m} J_{m(n-1)}+J_{m(n-2)}
$$




$$
\begin{aligned}
& =\left(\frac{a^{-\left\lfloor\frac{m+n}{2}\right\rfloor+\left\lfloor\frac{n}{2}\right\rfloor-\left[\frac{m(n-1)-1}{2}\right]_{b}\left\lfloor\frac{m+n}{2}\right\rfloor-\left\lfloor\frac{n}{2}\right\rfloor-\left\lfloor\frac{m}{2}\right\rfloor-\left\lfloor\frac{m+1}{2}\right\rfloor-\left\lfloor\frac{m(n-1)}{2}\right\rfloor}}{\alpha-\beta}\left(\alpha^{m}+\beta^{m}\right)\left(\alpha^{m(n-1)}-\beta^{m(n-1)}\right)\right)+ \\
& (-2)^{m+1}\left(\frac{a^{-\left\lfloor\frac{m(n-2)-1}{2}\right\rfloor_{b}-\left\lfloor\frac{m(n-2)}{2}\right\rfloor}}{\alpha-\beta}\left(\alpha^{m(n-2)}-\beta^{m(n-2)}\right)\right)
\end{aligned}
$$

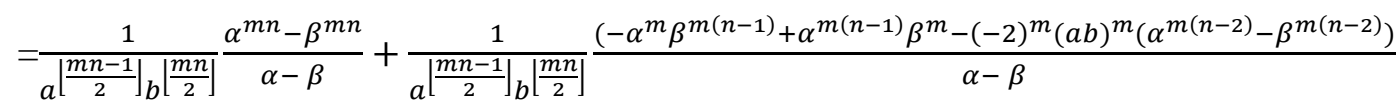

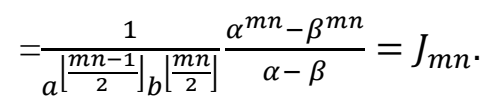

Now taking $a=b=1$ into Theorem 2.6 gives

$j_{m n}=j_{m(n-1)}-(-2)^{m} j_{m(n-2)}$.

Theorem 2.7 For any $n$, we have the following identities for bi-periodic Jacobsthal sequence and bi-periodic Jacobsthal Lucas sequence:

$J_{4 n+1}-2^{2 n}=\left(\frac{b}{a}\right) C_{2 n+1} J_{2 n}$

and

$$
J_{4 n+3}-2^{2 n+1}=\left(\frac{b}{a}\right) C_{2 n+1} J_{2 n+2} .
$$

Proof: (1)

$$
\begin{aligned}
C_{2 n+1} J_{2 n} & =\left(\frac{a^{\xi(2 n+1)}}{(a b)^{\left\lfloor\frac{2 n+2}{2}\right\rfloor}}\left(\alpha^{2 n+1}+\beta^{2 n+1}\right)\right)\left(\frac{a^{1-\xi(2 n)}}{\left.(a b)^{2 n} \frac{2 n}{2}\right]} \frac{\alpha^{2 n}-\beta^{2 n}}{\alpha-\beta}\right) \\
& =\frac{a^{2}}{(a b)^{2 n+1}}\left(\frac{\alpha^{4 n+1}-\beta^{4 n+1}-\alpha^{2 n+1} \beta^{2 n}+\beta^{2 n+1} \alpha^{2 n}}{\alpha-\beta}\right) \\
& =\frac{a^{2}}{(a b)^{2 n+1}}\left(\frac{\alpha^{4 n+1}-\beta^{4 n+1}-\alpha^{2 n} \beta^{2 n}(\alpha-\beta)}{\alpha-\beta}\right) \\
& =\frac{a}{b}\left[\frac{1}{(a b)^{2 n}} \frac{\alpha^{4 n+1}-\beta^{4 n+1}}{\alpha-\beta}\right]-\frac{a}{b} \frac{1}{(a b)^{2 n}}(a b)^{2 n}(-2)^{2 n} \\
& =\frac{a}{b}\left[\frac{1}{(a b)^{2 n}} \frac{\alpha^{4 n+1}-\beta^{4 n+1}}{\alpha-\beta}\right]-\frac{a}{b} \frac{1}{(a b)^{2 n}}(a b)^{2 n}(-2)^{2 n} \\
& =\frac{a}{b}\left[\frac{a^{1-\xi(4 n+1)}}{(a b)^{4 n+1}} \frac{\alpha^{4 n+1}-\beta^{4 n+1}}{\alpha-\beta}\right]-\frac{a}{b} 2^{2 n} \\
& =\frac{a}{b} J_{4 n+1}-\frac{a}{b} 2^{2 n}
\end{aligned}
$$

(2)

$$
\begin{aligned}
C_{2 n+1} J_{2 n+2} & =\left(\frac{a^{\xi(2 n+1)}}{(a b)^{\left\lfloor\frac{2 n+2}{2}\right\rfloor}}\left(\alpha^{2 n+1}+\beta^{2 n+1}\right)\right)\left(\frac{a^{1-\xi(2 n+2)}}{(a b)^{\left.\mid \frac{2 n+2}{2}\right\rfloor}} \frac{\alpha^{2 n+2}-\beta^{2 n+2}}{\alpha-\beta}\right) \\
& =\frac{a^{2}}{(a b)^{2 n+2}}\left(\frac{\alpha^{4 n+3}-\beta^{4 n+3}+\alpha^{2 n+1} \beta^{2 n+1}(\alpha-\beta)}{\alpha-\beta}\right)
\end{aligned}
$$




$$
\begin{aligned}
& =\frac{a}{b} \frac{a b}{(a b)^{2 n+2}} \frac{\alpha^{4 n+3}-\beta^{4 n+3}}{\alpha-\beta}+\frac{a}{b} \frac{a b}{(a b)^{2 n+2}} \frac{(\alpha \beta)^{2 n+1}(\alpha-\beta)}{\alpha-\beta} \\
& =\frac{a}{b} \frac{1}{(a b)^{2 n+1}} \frac{\alpha^{4 n+3}-\beta^{4 n+3}}{\alpha-\beta}+\frac{a}{b} \frac{1}{(a b)^{2 n+1}}(-2 a b)^{2 n+1} \\
& =\frac{a}{b} \frac{a^{1-\xi(4 n+3)}}{(a b)^{\left(\frac{4 n+3}{2}\right]}} \frac{\alpha^{4 n+3}-\beta^{4 n+3}}{\alpha-\beta}+\frac{a}{b}(-2)^{2 n+1} \\
& =\frac{a}{b} J_{4 n+3}-\frac{a}{b} 2^{2 n+1} .
\end{aligned}
$$

Now if we take $a=b=1$, we obtain the following

$c_{4 n+1}-(-2)^{2 n}=c_{2 n+1} j_{2 n}$

and

$c_{4 n+3}-(-2)^{2 n+1}=c_{2 n+1} j_{2 n+2}$.

Theorem 2.8 For any integer $n$, the identity for bi-periodic Jacobsthal sequence and bi-periodic Jacobsthal Lucas sequence is denoted by

$C_{n} C_{n+2}-(a b+8) J_{n-1} J_{n+3}=(-2)^{n-1} a^{1+\xi(n)} b^{\xi(n+1)}(a b+6)$

\section{Proof:}

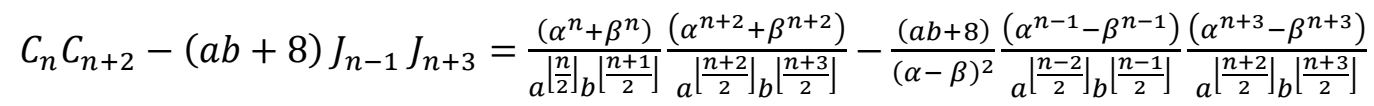

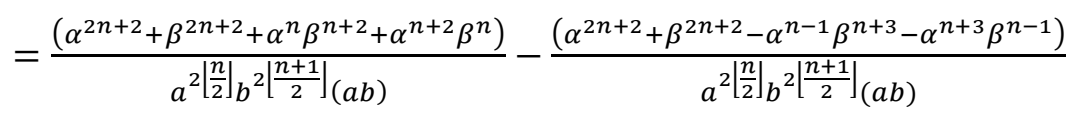

$$
\begin{aligned}
& =\frac{\alpha^{2 n+2}+\beta^{2 n+2}+\alpha^{n} \beta^{n}\left(\beta^{2}+\alpha^{2}\right)-\left[\alpha^{2 n+2}+\beta^{2 n+2}-\alpha^{n} \beta^{n}\left(\frac{\beta^{3}}{\alpha}+\frac{\alpha^{3}}{\beta}\right)\right]}{\left.a^{2}\left\lfloor\frac{n}{2}\right\rfloor b^{2\left\lfloor\frac{n+1}{2}\right.}\right\rfloor(a b)}
\end{aligned}
$$

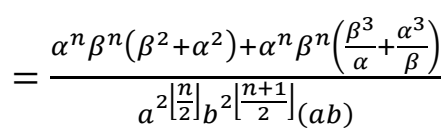

$$
\begin{aligned}
& =\frac{\alpha^{n} \beta^{n}\left(\beta^{2}+\alpha^{2}+\frac{\beta^{3}}{\alpha}+\frac{\alpha^{3}}{\beta}\right)}{a^{2\left\lfloor\frac{n}{2}\right\rfloor} b^{2\left\lfloor\frac{n+1}{2}\right\rfloor}(a b)} \\
& =\frac{\alpha^{n} \beta^{n}\left[\beta^{2}\left(1+\frac{\beta}{\alpha}\right)+\alpha^{2}\left(1+\frac{\alpha}{\beta}\right)\right]}{a^{2\left\lfloor\frac{n}{2}\right\rfloor_{b}{ }^{2}\left[\frac{n+1}{2}\right]}(a b)} \\
& =\frac{\alpha^{n} \beta^{n}\left[(\alpha+\beta)\left(\frac{\beta^{2}}{\alpha}+\frac{\alpha^{2}}{\beta}\right)\right]}{a^{2\left\lfloor\frac{n}{2}\right\rfloor} b^{2\left\lfloor\frac{n+1}{2}\right\rfloor}(a b)} \\
& =\frac{\alpha^{n} \beta^{n}\left[(\alpha+\beta)\left(\frac{\beta^{3}+\alpha^{3}}{\alpha \beta}\right)\right]}{a^{2\left\lfloor\frac{n}{2}\right\rfloor} b^{2\left\lfloor\frac{n+1}{2}\right\rfloor}(a b)}
\end{aligned}
$$




$$
\begin{aligned}
& =\frac{(\alpha \beta)^{n}\left[\frac{a b}{-2}\left[(a b)^{2}+6 a b\right]\right]}{a^{2}\left[\frac{n}{2}\right\rfloor b^{2}\left\lfloor\frac{n+1}{2}\right\rfloor(a b)} \\
& =\frac{a^{2} b^{2}}{-2} \frac{(-2 a b)^{n}(a b+6)}{a^{2}\left[\frac{n}{2} b^{2}\left[\frac{n+1}{2}\right\rfloor(a b)\right.} \\
& =\frac{(-2)^{n}}{-2} \frac{(a b)^{n+1}(a b+6)}{a^{2\left\lfloor\frac{n}{2}\right\rfloor} b^{2\left\lfloor\frac{n+1}{2}\right]}} \\
& =(-2)^{n-1} a^{1+n-2\left\lfloor\frac{n}{2}\right\rfloor} b^{n+1-2\left\lfloor\frac{n+1}{2}\right\rfloor}(a b+6) \\
& =(-2)^{n-1} a^{1+\xi(n)} b^{\xi(n+1)}(a b+6) .
\end{aligned}
$$

Now if we take $a=b=1$, we obtain the following

$c_{n} c_{n+2}-9 j_{n-1} j_{n+3}=7(-2)^{n-1}$.

Theorem 2.9 For any integer $m, n \geq 2$, the relation between bi-periodic Jacobsthal sequence and bi-periodic Jacobsthal Lucas sequence is given as

$J_{m} J_{n}-4 J_{m-2} J_{n-2}=a^{1-\xi(m n)} b^{\xi(m n)} J_{m+n-2}$

Proof: By $(1,2)$, we get

$J_{m} J_{n}=\frac{a^{2-\xi(m)-\xi(n)}\left[\alpha^{m+n}+\beta^{m+n}-\left(\alpha^{n} \beta^{m}+\alpha^{m} \beta^{n}\right)\right]}{(a b)^{\left[\frac{m}{2}\right\rfloor+\left[\frac{n}{2}\right\rfloor}(\alpha-\beta)^{2}}$

$J_{m-2} J_{n-2}=\frac{a^{2-\xi(m)-\xi(n)}(a b)^{2}\left[\alpha^{m+n-4}+\beta^{m+n-4}-(\alpha \beta)^{-2}\left(\alpha^{n} \beta^{m}+\alpha^{m} \beta^{n}\right)\right]}{(a b)^{\left\lfloor\frac{m}{2}\right]+\left[\frac{n}{2}\right]}(\alpha-\beta)^{2}}$

Then we subtract the equalities

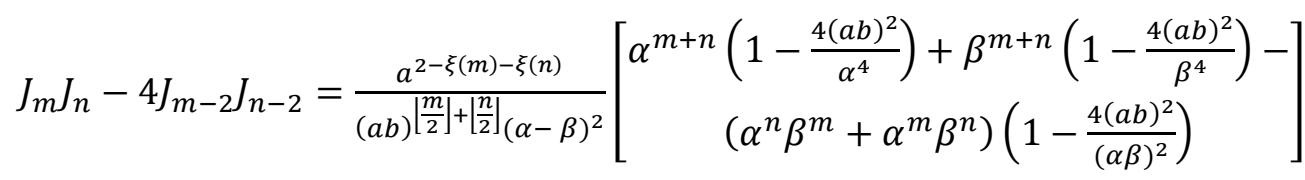

By $\alpha \beta=-2 a b$ and $\xi(m)+\xi(n)-\xi(m n)=\xi(m+n)$, we get

$$
\begin{aligned}
J_{m} J_{n}-4 J_{m-2} J_{n-2} & =\frac{a^{2-\xi(m)-\xi(n)}(\alpha+\beta)}{(a b)^{\left\lfloor\frac{m}{2}\right\rfloor+\left\lfloor\frac{n}{2}\right\rfloor}(\alpha-\beta)}\left(\alpha^{m+n-2}-\beta^{m+n-2}\right) \\
& =\frac{a^{2-\xi(m n)-\xi(m+n)}}{(a b)^{\left\lfloor\frac{m}{2}\right\rfloor+\left\lfloor\frac{n}{2}\right\rfloor-1}(\alpha-\beta)}\left(\alpha^{m+n-2}-\beta^{m+n-2}\right) \\
& =a^{1-\xi(m n)} \frac{a^{1-\xi(m+n-2)}}{(a b)^{\left\lfloor\frac{m}{2}\right\rfloor+\left[\frac{n-2}{2}\right\rfloor}(\alpha-\beta)}\left(\alpha^{m+n-2}-\beta^{m+n-2}\right) \\
& =a^{1-\xi(m n)} b^{\xi(m n)} \frac{a^{1-\xi(m+n-2)}}{(a b)^{\left\lfloor\frac{m+n-2}{2}\right\rfloor}}\left(\alpha_{(\alpha-\beta)}^{m+n-2}-\beta^{m+n-2}\right) \\
& =a^{1-\xi(m n)} b^{\xi(m n)} J_{m+n-2}
\end{aligned}
$$

Therefore, the proof is completed. 
Now if we take $a=b=1$, we obtain the following

$j_{m} j_{n}-4 j_{m-2} j_{n-2}=j_{m+n-2}$.

Theorem 2.10 A summation formula of bi-periodic Jacobsthal sequence with even index term is given by $J_{2 m}=-(a b+4)^{m-1}\left[-a+4 \sum_{k=0}^{m-2}(a b+4)^{-k-1} J_{2 k}\right]$.

\section{Proof:}

$$
\begin{aligned}
\sum_{k=0}^{m-2}(a b+4)^{-k-1} J_{2 k} & =\sum_{k=0}^{m-2}(a b+4)^{-k-1} \frac{a^{1-\xi(2 k)}}{(a b)^{2 k}}\left(\frac{\alpha^{2 k}-\beta^{2 k}}{\alpha-\beta}\right) \\
& =\frac{a}{(a b+4)(\alpha-\beta)} \sum_{k=0}^{m-2} \frac{\alpha^{2 k}-\beta^{2 k}}{(a b+4)^{k}(a b)^{k}} \\
& =\frac{a}{(a b+4)(\alpha-\beta)} \sum_{k=0}^{m-2}\left[\left(\frac{\alpha^{2}}{a b(a b+4)}\right)^{k}-\left(\frac{\beta^{2}}{a b(a b+4)}\right)^{k}\right] \\
& =\frac{a}{(a b+4)(\alpha-\beta)}\left(\frac{\left(\frac{\alpha^{2}}{a b(a b+4)}\right)^{m-1}-1}{\frac{\alpha^{2}}{a b(a b+4)}-1}-\frac{\left(\frac{\beta^{2}}{a b(a b+4)}\right)^{m-1}-1}{\frac{\beta^{2}}{a b(a b+4)}-1}\right) \\
& =\frac{a}{(a b+4)(\alpha-\beta)}\left(\begin{array}{l}
\frac{\alpha^{2 m-2}-(a b)^{m-1}(a b+4)^{m-1}}{\left(\alpha^{2}-a b(a b+4)\right)(a b)^{m-2}(a b+4)^{m-2}} \\
-\frac{\beta^{2 m-2}-(a b)^{m-1}(a b+4)^{m-1}}{\left(\beta^{2}-a b(a b+4)\right)(a b)^{m-2}(a b+4)^{m-2}}
\end{array}\right) \\
& =\frac{a}{(a b+4)^{m-1}(a b)^{m-2}(\alpha-\beta)}\left(\begin{array}{c}
\frac{\alpha^{2 m-2}-(a b)^{m-1}(a b+4)^{m-1}}{\alpha^{2}-a b(a b+4)} \\
-\frac{\beta^{2 m-2}-(a b)^{m-1}(a b+4)^{m-1}}{\beta^{2}-a b(a b+4)}
\end{array}\right)
\end{aligned}
$$

It is noticed that,

$$
\begin{aligned}
& \sum_{k=0}^{m-2}(a b+4)^{-k-1} J_{2 k}=\frac{a}{(a b+4)^{m-1}(a b)^{m-2}(\alpha-\beta)}\left(\frac{\begin{array}{c}
4(a b)^{2}\left(\alpha^{2 m-4}-\beta^{2 m-4}\right)-a b(a b+4)\left(\alpha^{2 m-2}-\beta^{2 m-2}\right) \\
+(a b)^{m-1}(a b+4)^{m-1}\left(\alpha^{2}-\beta^{2}\right)
\end{array}}{4(a b)^{2}}\right) \\
& =\frac{a\left(\alpha^{2 m-4}-\beta^{2 m-4}\right)}{(a b+4)^{m-1}(a b)^{m-2}(\alpha-\beta)}-\frac{a\left(\alpha^{2 m-2}-\beta^{2 m-2}\right)}{4(a b+4)^{m-2}(a b)^{m-1}(\alpha-\beta)}+\frac{a}{4} \\
& =\frac{J_{2 m-4}}{(a b+4)^{m-1}}-\frac{J_{2 m-2}}{4(a b+4)^{m-2}}+\frac{a}{4} \\
& =\frac{4 J_{2 m-4}-(a b+4) J_{2 m-2}}{4(a b+4)^{m-1}}+\frac{a}{4}
\end{aligned}
$$

Consequently,

$$
\sum_{k=0}^{m-2}(a b+4)^{-k-1} J_{2 k}=-\frac{1}{4(a b+4)^{m-1}} J_{2 m}+\frac{a}{4}
$$

The proof is completed.

Now if we take $a=b=1$, we obtain the following

$j_{2 m}=-5^{m-1}\left[-a+4 \sum_{k=0}^{m-2}(5)^{-k-1} j_{2 k}\right]$. 


\section{Acknowledgment}

I would like to acknowledge Prof. Dr. Baki KESKIN for technical and editorial assistance and referees for their valuable comments.

\section{Conflicts of interest}

The authors state that did not have a conflict of interests.

\section{References}

[1] Horadam A. F., Jacobsthal Representation Numbers, The Fibonacci Quarterly, 37 (2) (1996) 40-54.

[2] Edson M, Yayenie O., A New Generalization of Fibonacci Sequences and Extended Binet's Formula, Integers, 9 (2009) 639-654.

[3] Yayenie O., A Note on Generalized Fibonacci Sequence, Applied Mathematics and Computation, 217 (2011) 5603-5611.

[4] Jun S.P, Choi K.H., Some Properties of the Generalized Fibonacci Sequence $\left\{\mathrm{q}_{\mathrm{n}}\right\}$ by Matrix Methods, The Korean Journal of Mathematics, 24 (4) (2016) 681-691.

[5] Bilgici G, Two Generalizations of Lucas Sequence, Applied Mathematics and Computation, 245 (2014) 526-538.

[6] Uygun S., Owusu E., A New Generalization of Jacobsthal Numbers (Bi-Periodic Jacobsthal Sequences), Journal of Mathematical Analysis, 5 (2016) 728-39.

[7] Uygun S., Karatas H., Akınc1 E., Relations on Biperiodic Jacobsthal Sequence, Transylvanian Journal of Mathematics and Mechanics, 10 (2) (2018) 141-151.
[8] Uygun S., Owusu E., A Note on bi-periodic Jacobsthal Lucas Numbers, Journal of Advances in Mathematics and Computer Science, 34 (5) (2019) 1-13.

[9] Uygun S., Karatas H., A New Generalization of Pell-Lucas Numbers (Bi-Periodic Pell-Lucas Sequence), Communications in Mathematics and Applications, 10 (3) (2019) 1-12.

[10] Choo Y., Some Identities on Generalized Biperiodic Fibonacci Sequences, International Journal of Mathematical Analysis, 13 (6) (2019) 259-267.

[11] Gul K., On Bi-periodic Jacobsthal and JacobsthalLucas Quaternions, Journal of Mathematics Research, 11 (2) (2019) 44-52.

[12] Komatsu T., Ramírez J.L., Convolutions of the Bi-periodic Fibonacci Numbers, Hacettepe Journal of Mathematics \& Statistics, (2019) Early Access: 1-13.

[13] Brigham R., Chinn P., Grimaldi R., Tiling and Patterns of Enumeration, Congressus Numerantium, 137 (1999) 207-219.

[14] Frey D., Sellers J., Jacobsthal Numbers and Alternating Sign Matrices, Journal. of Integer Sequences, 3 (2000).

[15] Grimaldi R., Binary Strings and the Jacobsthal Numbers, Congressus Numerantium, 174 (2005) 3-22.

[16] Grimaldi R., The Distribution of 1's in Jacobsthal Binary Sequences, Congressus Numerantium, 190 (2008) 47-64. 